\title{
MTF DETERMINATION OF SENTINEL-4 DETECTOR ARRAYS
}

\author{
R. Reulke ${ }^{\text {a }}$ I. Sebastian ${ }^{\mathrm{b}}$, C. Williges ${ }^{\mathrm{b}}$, R. Hohn ${ }^{\mathrm{b}}$ \\ ${ }^{a}$ Department of Computer Science, Humboldt-Universitt zu Berlin, Germany - reulke@informatik.hu-berlin.de \\ ${ }^{b}$ Institut of Optical Sensor Systems, German Aerospace Center DLR, 12489 Berlin, Germany - (christian.williges, ilse.sebastian)@dlr.de \\ b Airbus Defense and Space, 85521 Ottobrunn, Germany - ruediger.hohn@airbus.com
}

\section{Commission I, WG I/10}

KEY WORDS: PSF measurement, MTF evaluation, CCD, FPA, Sentinel-4

\begin{abstract}
:
The Institute for Optical Sensor Systems was involved in many international space projects in recent years. These include, for example, the fokal plane array (FPA) of the hyperspectral sensors ENMAP or Sentinel-4, but also the FPA for the high resolution FPA for Kompsat-3. An important requirement of the customer is the measurement of the detector MTF for different wavelengths. A measuring station under clean room conditions and evaluation algorithms was developed for these measurements. The measurement setup consist of a collimator with slit target in focus for illumination at infinity, a gimbal mounted detector facing an auxiliary lens in front, a halogen lamp with monochromator or filter, as well as optical and electrical ground support equipment. Different targets and therefore also different measurement and data evaluation opportunities are possible with this setup. Examples are slit, edge, pin hole but also a Siemens star. The article describes the measurement setup, the different measuring and evaluation procedures and exemplary results for Sentinel-4 detector.
\end{abstract}

\section{INTRODUCTION}

The Sentinel-4 payload is a multi-spectral camera system which is designed to monitor atmospheric conditions over Europe. The German Aerospace Center (DLR) in Berlin, Germany conducted the verification campaign of the Focal Plane Subsystem (FPS) on behalf of Airbus Defense and Space GmbH, Ottobrunn, Germany. In this publication, we will present in detail the MTF measurement set-up and data evaluation for deriving MTF at Nyquist frequency for the CCD 374 (UVVIS I and II) as well as CCD376 (NIR). A description of these back side illumination (BSI) detectors can be found in the publication (Jerram and Morris, 2016).

In this article, we focus on the MTF for the highest (ca. $500 \mathrm{~nm}$ ) and lowest wavelengths (ca. $300 \mathrm{~nm}$ ) for the UVVIS detector. Because of subsurface diffusion effects we expect for BSI detectors the best results for the MTF at larger wavelengths.

The paper is organised as follows. First, a brief overview of the theory is given. The measurement setup is then described. In the next chapter the data evaluation and derivation of the MTF is described. Finally, a brief overview of the results is given. The paper comes up with conclusions and outlook.

\section{PSF AND MTF}

An overview of recent developments in scientific CCDs, in particular front- and back-site illuminated CCDs, and the characterization (including MTF measurements) of these devices can be found in (Lesser, 2015a) and (Lesser, 2015b). In (Swindells et al., 2014) an e2v imaging detector for the visible channel of the Euclid space telescope was described. This detector is an $22 \mathrm{v}$ back-illuminated, $4 \mathrm{k}$ x 4k, 12 micron square pixel CCD designated CCD273-84. Results are presented also for MTF measurements, which shows a slight improvement with wavelength.
An overview about PSF and MTF theory can be found in the book (Boreman, 2001) or (Jahn and Reulke, 2009). The PSF measurement is based on the definition of a translation invariant PSF:

$$
V(x, y)=\int_{-\infty}^{\infty} \int_{-\infty}^{\infty} d x^{\prime} d y^{\prime} H\left(x-x^{\prime}, y-y^{\prime}\right) \cdot U\left(x^{\prime}, y^{\prime}\right)
$$

The PSF can be calculated through the input signal $U(x, y)$ and the measurement of $V(x, y)$. Conceptually, this is the shape of the blurred image of a point source (i.e., the blur spot). Together with PSF also Line Spread Function (LSF) and Edge Spread Function (ESF) can be derived:

- PSF from response of a point-like object (delta-function)

$$
U\left(x^{\prime}, y^{\prime}\right)=\delta\left(x^{\prime}, y^{\prime}\right) \Rightarrow V(x, y)=H(x, y)
$$

- LSF from response of a line-like object (parallel to y-axis)

$$
U\left(x^{\prime}, y^{\prime}\right)=\delta\left(x^{\prime}, y^{\prime}\right) \Rightarrow V(x)=\int_{-\infty}^{\infty} d x^{\prime} H\left(x-x^{\prime}\right)
$$

- ESF from response of a black to white edge (parallel to yaxis)

$$
U\left(x^{\prime}, y^{\prime}\right)=\left\{\begin{array}{ll}
0 & x>0 \\
1 & x \leq 0
\end{array} \Rightarrow V(x)=\int_{-\infty}^{x} d x^{\prime} H\left(x-x^{\prime}\right)\right.
$$


In laboratory these three functions can be fully estimated with pinholes, tri-bars, slit or slanted edge (Reulke et al., 2011). As mentioned, Point Spread Function (PSF), Modulation Transfer Function (MTF) and Edge Spread Function (ESF) are mathematically related: The system MTF is the module of the Optical Transfer Function (OTF) derived as the 2D-Fourier Transform of the Point Spread Function and the derivative of the LSF with respect to position is Point Spread Function (PSF) in that direction. ESF or its normalized version of the Relative Edge Response (RER) and can be evaluated instead of the PSF or MTF. In real images the ESF from light to dark transitions was estimated using natural edges such as bridges or runways and it was assumed that the PSF had a normal distribution:

$$
H(x)=\frac{1}{\sigma_{H} \sqrt{2 \pi}} \cdot e^{-\frac{x}{2 \sigma_{H}^{2}}}
$$

Thus, a first quantitative and shape description of the PSF is given by the parameter $\sigma_{H}$. Assuming for the PSF to a Gaussian distribution, the ESF is an error function

$$
y=\frac{p_{0}}{2} \cdot\left(1+\operatorname{Erf}\left(\frac{x-p_{1}}{p_{2} \cdot \sqrt{2}}\right)\right)+p_{3}+x \cdot p_{4}
$$

With $\operatorname{Erf}(z)=\frac{2}{\sqrt{\pi}} \cdot \int_{0}^{z} e^{-t^{2}} d t$.

\section{MEASUREMENT SETUP}

\subsection{Theory}

In this section, the measurement setup is to be investigated theoretically more precisely.

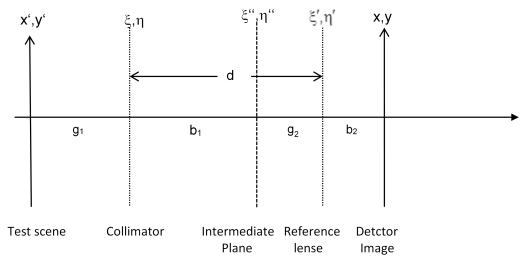

Figure 1. Measurement setup

Figure 1 shows the schematic sketch of setup. The test scene is imaged into the infinity by the collimator and focused on the detector by the reference lens. The collimator realise an image in the intermediate plane. From left to right we have the following amplitudes:

\section{Amplitude in front of the collimator}

$$
U_{\lambda}^{V}(\xi, \eta) \sim \iint d x^{\prime} d y^{\prime} U_{\lambda}^{E}\left(x^{\prime}, y^{\prime}\right) \cdot e^{i \cdot \frac{\pi}{\lambda \cdot g_{1}} \cdot\left[\left(\xi-x^{\prime}\right)^{2}+\left(\eta-y^{\prime}\right)^{2}\right]}
$$

\section{The amplitude in the intermediate plane is}

$$
U_{\lambda}^{Z}\left(\xi^{\prime \prime}, \eta^{\prime \prime}\right) \sim \iint d x^{\prime} d y^{\prime} H_{\lambda}^{(1)}\left(\xi^{\prime \prime}, \eta^{\prime \prime} ; x^{\prime}, y^{\prime}\right) \cdot U_{\lambda}^{E}\left(x^{\prime}, y^{\prime}\right)
$$

With the (amplitude) PSF of the collimator

$$
\begin{aligned}
& H_{\lambda}^{(1)}\left(\xi^{\prime \prime}, \eta^{\prime \prime} ; x^{\prime}, y^{\prime}\right) \sim e^{i \cdot \frac{\pi}{\lambda \cdot b_{1}} \cdot\left(\xi^{\prime \prime 2}+\eta^{\prime \prime 2}\right)} \cdot e^{i \cdot \frac{\pi}{\lambda \cdot g_{1}} \cdot\left(x^{\prime 2}+y^{\prime 2}\right)} . \\
& \quad \iint d \xi d \eta O_{1}(\xi, \eta) \cdot e^{i \cdot \frac{\pi}{\lambda} \cdot \varepsilon_{1} \cdot\left(\xi^{2}+\eta^{2}\right)} . \\
& \quad e^{-i \cdot \frac{2 \pi}{\lambda \cdot b_{1}} \cdot\left[\xi \cdot\left(\xi^{\prime \prime}+\frac{b_{1}}{g_{1}} \cdot x^{\prime}\right)+\eta \cdot\left(\eta^{\prime \prime}+\frac{b_{1}}{g_{1}} \cdot y^{\prime}\right)\right]} \\
& \left(\varepsilon_{1}=\frac{1}{g_{1}}+\frac{1}{b_{1}}-\frac{1}{f_{1}}\right)
\end{aligned}
$$

For incoherent radiation, we have

$$
\left\langle U_{\lambda}^{E}\left(x^{\prime}, y^{\prime}\right) \cdot U_{\lambda}^{E *}\left(x^{\prime \prime}, y^{\prime \prime}\right)\right\rangle=I_{\lambda}^{E}\left(x^{\prime}, y^{\prime}\right) \cdot \delta\left(x^{\prime}-x^{\prime \prime}\right) \cdot \delta\left(y^{\prime}-y^{\prime \prime}\right)
$$

$I_{\lambda}^{E}\left(x^{\prime}, y^{\prime}\right)$ is the intensity of the incoherent Input radiation. In the intermediate plane the intensity is

$$
h_{\lambda}^{(1)}\left(\xi^{\prime \prime}, \eta^{\prime \prime} ; x^{\prime}, y^{\prime}\right)=\left|H_{\lambda}^{(1)}\left(\xi^{\prime \prime}, \eta^{\prime \prime} ; x^{\prime}, y^{\prime}\right)\right|^{2}
$$

The (intensity) PSF of the collimator. The amplitude in the image plane is

$$
U_{\lambda}^{A}(x, y) \sim \iint d \xi^{\prime \prime} d \eta^{\prime \prime} H_{\lambda}^{(2)}\left(x, y ; \xi^{\prime \prime}, \eta^{\prime \prime}\right) \cdot U_{\lambda}^{Z}\left(\xi^{\prime \prime}, \eta^{\prime \prime}\right)
$$

With the (amplitude) PSF of the test lens:

$$
\begin{aligned}
& H_{\lambda}^{(2)}\left(x, y ; \xi^{\prime \prime}, \eta^{\prime \prime}\right) \sim e^{i \cdot \frac{\pi}{\lambda \cdot b_{2}} \cdot\left(x^{2}+y^{2}\right)} \cdot e^{i \cdot \frac{\pi}{\lambda \cdot g_{2}} \cdot\left(\xi^{\prime \prime 2}+\eta^{\prime \prime 2}\right) .} \\
& \quad \iint d \xi^{\prime} d \eta^{\prime} O_{2}\left(\xi^{\prime}, \eta^{\prime}\right) \cdot e^{i \cdot \frac{\pi}{\lambda} \cdot \varepsilon_{2} \cdot\left(\xi^{\prime 2}+\eta^{\prime 2}\right) .} \\
& \quad e^{-i \cdot \frac{2 \pi}{\lambda \cdot b_{2}} \cdot\left[\xi^{\prime} \cdot\left(x+\frac{b_{2}}{g_{2}} \cdot \xi^{\prime \prime}\right)+\eta^{\prime} \cdot\left(y+\frac{b_{2}}{g_{2}} \cdot \eta^{\prime \prime}\right)\right]} \\
& \left(\varepsilon_{2}=\frac{1}{g_{2}}+\frac{1}{b_{2}}-\frac{1}{f_{2}}\right)
\end{aligned}
$$

For incoherent radiation we have

$$
I_{\lambda}^{A}(x, y) \sim \iint d \xi^{\prime \prime} d \eta^{\prime \prime} h_{\lambda}^{(2)}\left(x, y ; \xi^{\prime \prime}, \eta^{\prime \prime}\right) \cdot I_{\lambda}^{Z}\left(\xi^{\prime \prime}, \eta^{\prime \prime}\right)
$$

With

$$
h_{\lambda}^{(2)}\left(x, y ; \xi^{\prime \prime}, \eta^{\prime \prime}\right)=\left|H_{\lambda}^{(2)}\left(x, y ; \xi^{\prime \prime}, \eta^{\prime \prime}\right)\right|^{2}
$$

The (intensity) PSF of the second optics. This is valid for an isolated subsystem 2 and with incoherent radiation. In our optical system, however, the radiation in the intermediate plane is not incoherent, so upper equation is not correct here. For the whole optical system of collimator and reference lens we use the following equation:

$$
U_{\lambda}^{A}(x, y) \sim \iint d x^{\prime} d y^{\prime} H_{\lambda}^{G}\left(x, y ; x^{\prime}, y^{\prime}\right) \cdot U_{\lambda}^{E}\left(x^{\prime}, y^{\prime}\right)
$$

with

$$
H_{\lambda}^{G}\left(x, y ; x^{\prime}, y^{\prime}\right) \sim \iint d \xi^{\prime \prime} d \eta^{\prime \prime} H_{\lambda}^{(2)}\left(x, y ; \xi^{\prime \prime}, \eta^{\prime \prime}\right) \cdot H_{\lambda}^{(1)}\left(\xi^{\prime \prime}, \eta^{\prime \prime} ; x^{\prime}, y^{\prime}\right)
$$


For intensities we use the following equation

$$
I_{\lambda}^{A}(x, y) \sim \iint d x^{\prime} d y^{\prime} h_{\lambda}^{G}\left(x, y ; x^{\prime}, y^{\prime}\right) \cdot I_{\lambda}^{E}\left(x^{\prime}, y^{\prime}\right)
$$

with

$$
\begin{aligned}
& h_{\lambda}^{G}\left(x, y ; x^{\prime}, y^{\prime}\right)=\left|H_{\lambda}^{G}\left(x, y ; x^{\prime}, y^{\prime}\right)\right|^{2}= \\
& \left|\iint d \xi^{\prime \prime} d \eta^{\prime \prime} H_{\lambda}^{(2)}\left(x, y ; \xi^{\prime \prime}, \eta^{\prime \prime}\right) \cdot H_{\lambda}^{(1)}\left(\xi^{\prime \prime}, \eta^{\prime \prime} ; x^{\prime}, y^{\prime}\right)\right|^{2}
\end{aligned}
$$

This is the (intensity) PSF of the entire system. It can not be represented as a convolution of two partial PSFs.

Therefore, we work with the following equation:

$$
\begin{aligned}
& \hat{I}_{\lambda}^{A}(x, y) \sim \\
& {\left[\iint d x^{\prime} d y^{\prime} h_{\lambda}^{(2)}\left(x, y ; \xi^{\prime \prime}, \eta^{\prime \prime}\right) \cdot h_{\lambda}^{(1)}\left(\xi^{\prime \prime}, \eta^{\prime \prime} ; x^{\prime}, y^{\prime}\right)\right] .} \\
& \quad I_{\lambda}^{E}\left(x^{\prime}, y^{\prime}\right)
\end{aligned}
$$

Here the initial intensity is characterised by a hat for clarifying the incorrect calculation. From 20 one obtains the (incorrect) PSF of the optical overall system:

$$
\begin{aligned}
& \hat{h}_{\lambda}^{G}\left(x, y ; x^{\prime}, y^{\prime}\right)=\iint d \xi^{\prime \prime} d \eta^{\prime \prime} \\
& h_{\lambda}^{(2)}\left(x, y ; \xi^{\prime \prime}, \eta^{\prime \prime}\right) \cdot h_{\lambda}^{(1)}\left(\xi^{\prime \prime}, \eta^{\prime \prime} ; x^{\prime}, y^{\prime}\right)
\end{aligned}
$$

The result of the calculation is, that large collimator aperture the result is independent from spatial frequency. Assuring that a sufficiently large aperture of the collimator is realised, the total MTF is determined only by the test lens. The collimator then has no effect on the MTF of the whole system.

\subsection{Experimental Setup}

The Optical Ground Support Equipment (OGSE) to be used for MTF measurement is located in a clean room class 100000 . The common set-up of CCD and the auxiliary lens optics Flektogon 2.8/20 facing the collimator has been illuminated by a slit at infinity. The auxilary lens and the FPA are mounted together on a gimbal. Selected adjustment steps have to be performed to adjust the lens in a best focus position per wavelength in front of the CCD.

The collimator has a focal length of $1200 \mathrm{~mm}$ with an aperture diameter of $150 \mathrm{~mm}$. The lens optics has a focal length of $20 \mathrm{~mm}$ and an aperture diameter of $10 \mathrm{~mm}$. Due to the large ratio between the two focal lengths, a displacement in front of the collimator in the $m m$ range causes a displacement of the test object in front of the test object in the $\mu m$ range. The slit width and length in the image plane is $8.7 \mu m \times 130 \mu m$. Therefore we have approximately 8 pixel in horizontal and about 5 pixel in vertical direction. With the short length of the slit, the derivation of the MTF is actually a 2D problem. However, one can start from the analysis of a 1D problem and evaluate the line spread function. In Figure 2 the signal in slit direction for a short slit is shown. In our case, we have a $\sigma \approx 0.5$. In this case the influence of the $2 \mathrm{D}$ problem is in the middle of the slit negligible and we can work with the $1 \mathrm{D}$ case.

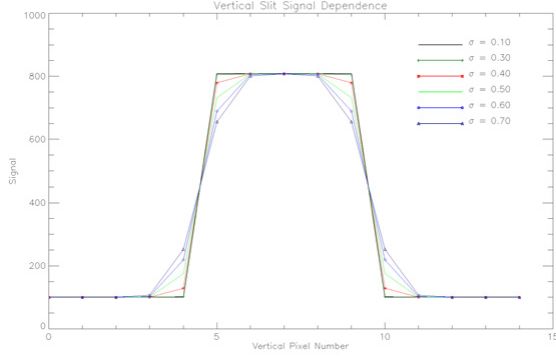

Figure 2. Signal in slit direction for a short slit. The influence of the $2 \mathrm{D}$ problem is in the middle of the slit negligible

The slit target with geometric slit width of about $1 / 10$ of a CCD pixel width will be located in collimator focus. The focus position can be shifted by $\mathrm{x}$ - and $\mathrm{y}$ - step motors. The wavelength selection will be done by selecting a metal interference filter that is arranged between halogen lamp and collimator. Figure 3 illustrates this set-up.

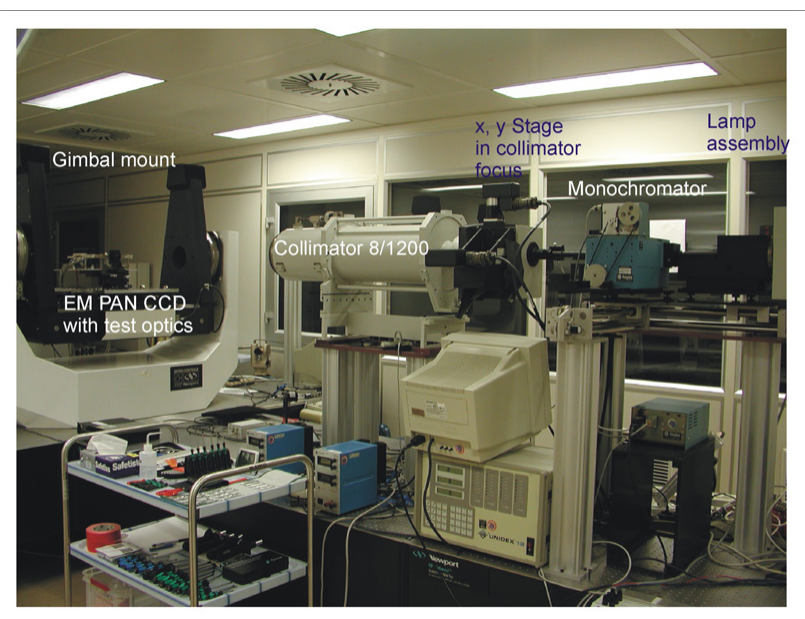

Figure 3. DLR Optical Ground Support Equipment

\subsection{Lens Optics Flektogon 2.8/20 MTF measured by Schnei- der Kreuznach}

Figure 4 shows the on-axis radial and tangential spectral MTFs of the Flektogon 2.8/20 measured by Schneider Kreuznach Optische Werke $\mathrm{GmbH}$ at $501 \mathrm{~nm}, 671 \mathrm{~nm}$ and $880 \mathrm{~nm}$. For each spectral MTF measurement the individual best focus position has been adjusted in accordance with the dedicated procedure. The MTF curves will be used for MTF correction in a later step.

\subsection{Detector}

We investigate the Sentinel-4 CCD 374 (UVVIS I and II) detector. The silicon photosensitive area consists of a split frame transfer CCD with two image zones. Both image zones have independent readout registers, different spectral ranges and integration times. The CCD has the following features: 


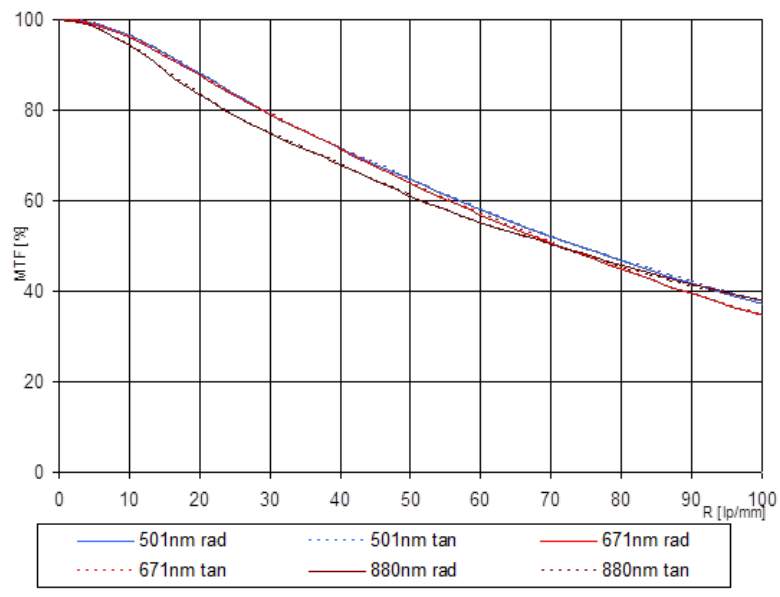

Figure 4. Spectral on-axis MTFs of the Flektogon 2.8/20 measured by Schneider Kreuznach

\section{UVVIS Detector}

Spectral range:

Pixel size

With

- graded AR coating

- thinned

- back side illuminated

- no antiblooming capabilities

\section{UVVIS I (Zone I)}

Spectral range:

Split into:

UVVISIa:

UVVISIb:

With

- two outputs

- different gains

- active pixels: (see Figure 5)

$305 \mathrm{~nm}-500 \mathrm{~nm}$

(spatial): $27.5 \mu \mathrm{m}$

(spectral): $15.0 \mu \mathrm{m}$

\section{UVVIS II (Zone II)}

Spectral range:

With

- four outputs

- active pixels:

\author{
$305 n m-343 n m$ \\ $305 \mathrm{~nm}-316.5 \mathrm{~nm}$ \\ $316.5 \mathrm{~nm}-343 \mathrm{~nm}$ \\ (used alternately) \\ 584pixels $\times 255$ pixels
}

$343 n m-500 n m$

584 pixels $\times 1025$ pixels

\subsection{Measurement Procedure}

Figure 6 illustrates the principle of the measurement approach for the Sentinel-4 CCD. The slit image spot at infinity will be positioned perpendicular to CCD line or column direction. Single Pixel Illumination of a selected centre pixel is performed by measurement of signal at a certain pixel vs. slit step position.

The signal distribution for a certain pixel position is shown in Figure 7. The slit length is about 8 pixel in vertical direction and 5 pixel horizontal direction. At each pixel position at least 10 measurements are available, which are related to a known slit position.

In order to analyse signal stability, the signal was measured approximately 30 times for each slit position (see Figure 8). The figure shows that the time dependency has no visible drift. Mean

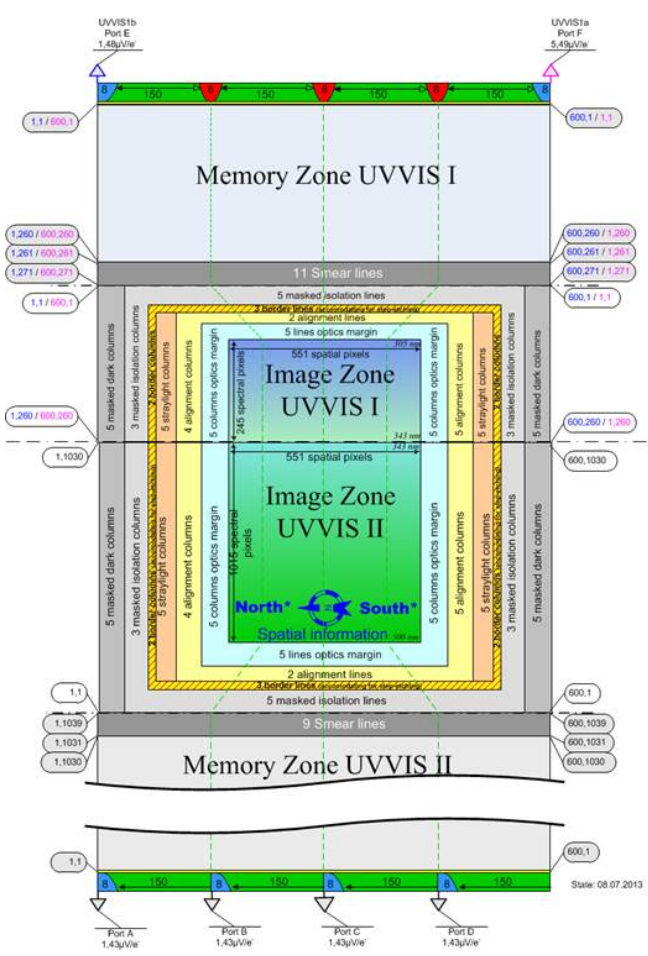

Figure 5. UVVISII detector

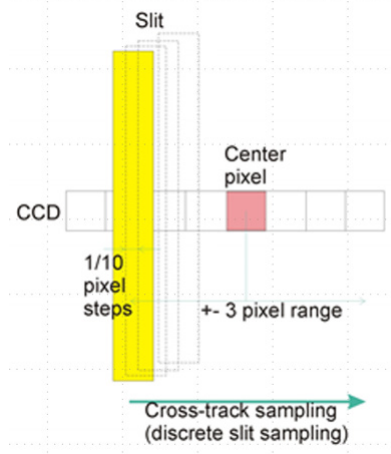

Figure 6. Measurement Procedure

and standard deviation for each measured value at different pixel positions is shown in Figure 9.

In Figure 9 the signal distribution, measured on different single pixel, is evaluated later for MTF determination. The error bars were also plotted. It can be seen clearly that the standard deviation of the measured signals is very small.

In the Figure 10 the variation of the signal from 77 measurements (slit steps) was analysed for different pixel around a predefined centre pixel in spatial and spectral direction. The PRNU of the detector, but in particular the spatial variation of the illumination, is responsible for the large change in the amplitude.

\subsection{Data Evaluation}

In the following, the signal is analysed in horizontal and vertical direction for a single pixel. The step width of the slit is predefined and is selected such that approximately 10 steps per pixel are shifted (in spectral $2.5 \mu \mathrm{m}$ and in spatial direction $1.358 \mu \mathrm{m}$ ). 


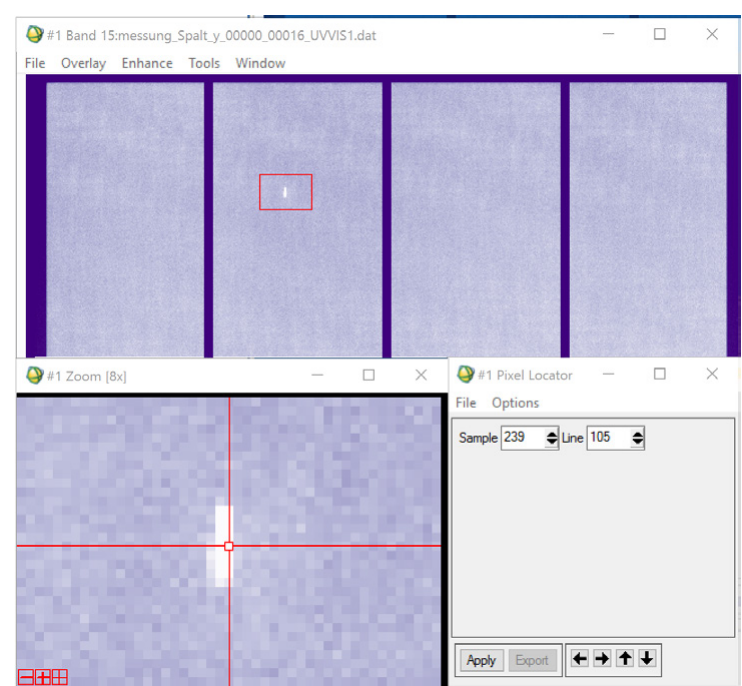

Figure 7. Signal distribution over the UVVISI detector with slit in vertical direction

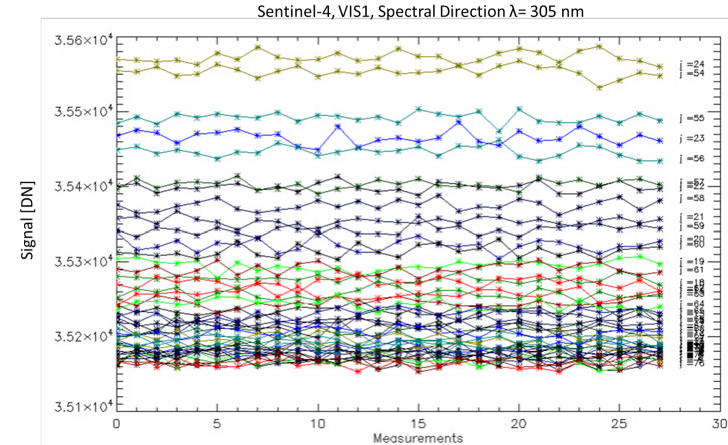

Figure 8. Signal over time for different slit positions

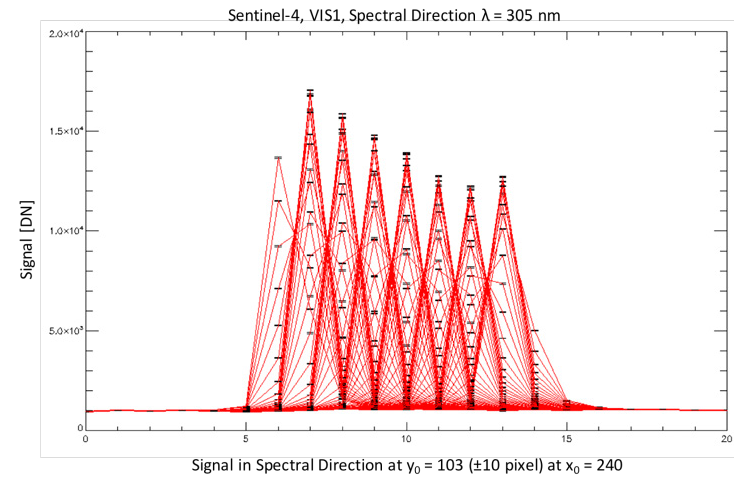

Figure 9. Signal at different slit positions

Figure 11 shows the signal in dependence of pixel position. We have also analysed the 1-sigma uncertainty of the signal. From the illustration it becomes clear that the drift and noise is negligible.

For the further data evaluation the zero point correction is necessary (see green line in Figure 11). The waveform close to the maximum of the signal differs significantly between the two spectral channels. The distinct structure in the long-wave spectral range has effects on the frequency response of the MTF above

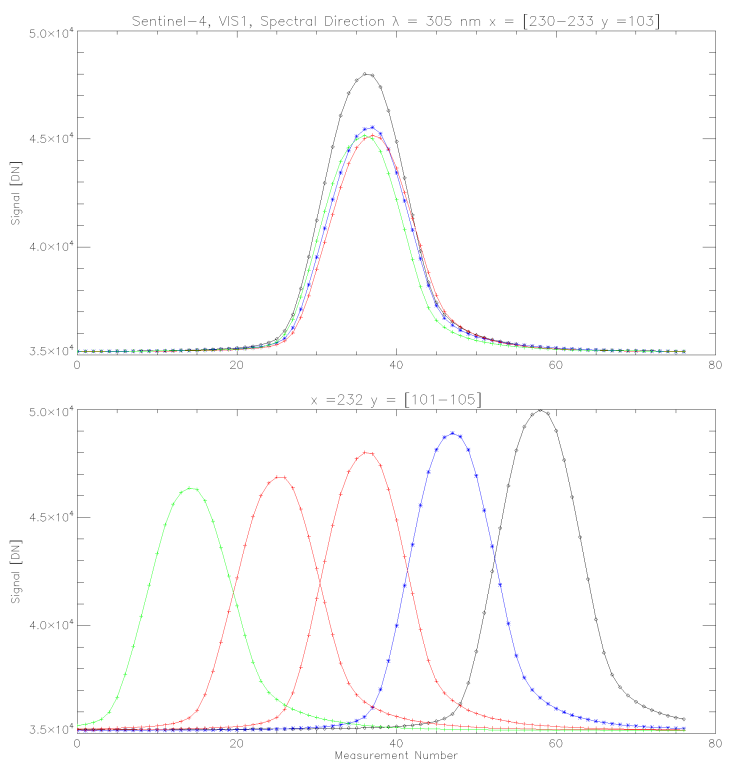

Figure 10. Spectral PSF: In horizontal or spatial direction (upper image) and in vertical or spectral direction (lower image).

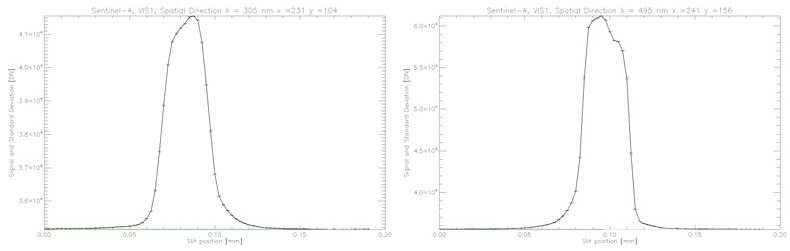

Figure 11. Signal distribution over a pixel $(305 \mathrm{~nm}$ left, $495 \mathrm{~nm}$ right). Green line is a zero-point correction

the first zero of the sinc function.

\section{RESULTS}

The evaluation was carried out in a region of $3 \times 3$ pixels around the centre pixel. In figure 12 the MTF from this 9 pixel are plotted. The thick green line is the expected MTF shape, derived from the optics-, slit- and pixel MTF. It is clear from Frigure 12 that the influences of pixel size, optics and slit size are sufficient for describing the measurement signal. The 9 measurements also show that the MTF variation is in a few percent range.

In Figure 13 the correction by optics and slit MTF of the MTF is visible in the spatial and spectral directions. The results are close to the physical limit of the pixels MTF (63\%). In addition, error bars were plotted in order to characterise the standard deviation of the individual measuring points.

The results are summarised below. For this purpose, the MTF was analysed as a function of the signal (see Table 1) and the wavelength (Table 2). (In the table, earlier studies were also included.)

It is shown that the MTF is independent of the signal. In addition, the MTF is about $2-3 \%$ smaller in the spatial direction than in the spectral direction. The spectral dependence of the MTF can not be reliably demonstrated. 


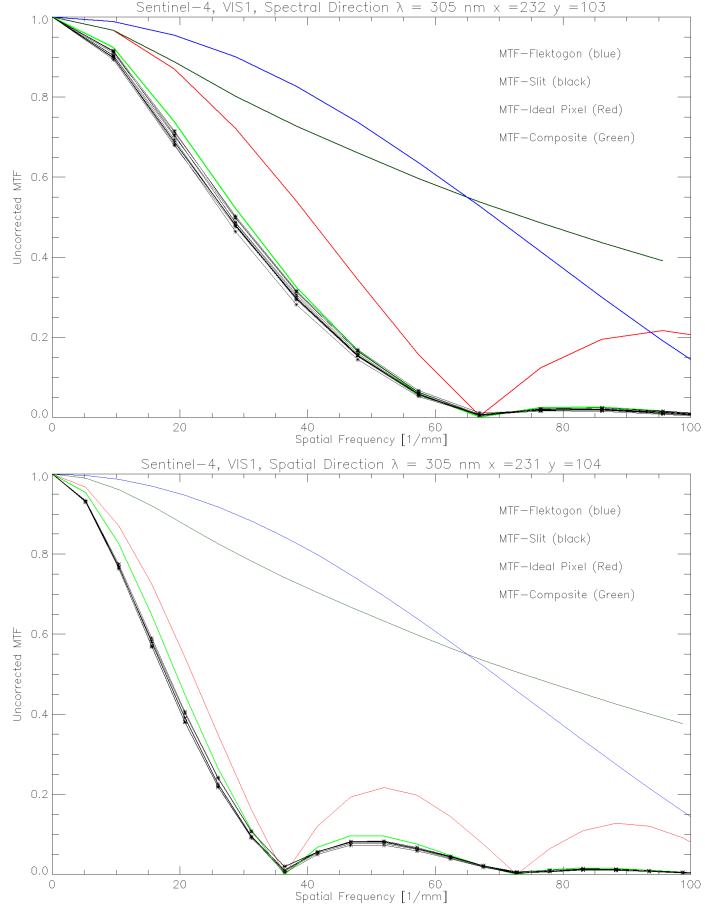

Figure 12. Uncorrected MTF (at 305 $\mathrm{nm}$ ) in comparison to theoretical influences. Spectral diection (upper image, $15 \mu \mathrm{m}$ ) and spatial direction (lower image $27.5 \mu \mathrm{m}$ ).

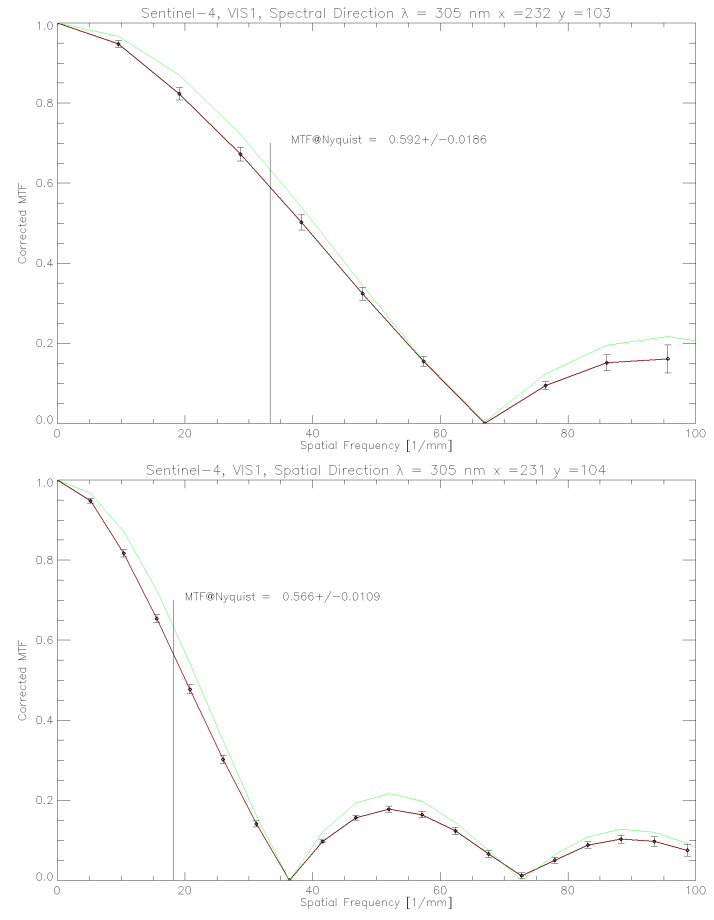

Figure 13. MTF (at 305 $\mathrm{nm}$ ) in spectral (upper image, $15 \mu \mathrm{m}$ ) and spatial direction (lower image $27.5 \mu \mathrm{m}$ )

\section{CONCLUSION AND OUTLOOK}

In the paper, a generic approach for the determination of MTF of detectors with a slit function was presented. The operability of the approach was shown by means of some examples. It has been shown that the derived MTF is within the expected range.

\begin{tabular}{l|l|l} 
Signal & MTF (mean) & MTF (Std.dev) \\
\hline 8TDN & $61.9 \%$ & $0.7 \%$ \\
14TDN & $61.3 \%$ & $0.6 \%$ \\
60TDN & $60.9 \%$ & $0.6 \%$
\end{tabular}

Table 1. Signal dependence (spatial direction, $495 \mathrm{~nm}$ ) at Nyquist Frequency

\begin{tabular}{l|l|l|l|l}
$\begin{array}{l}\text { Wave- } \\
\text { lenght }\end{array}$ & $\begin{array}{l}\text { Spectral } \\
\text { (mean) }\end{array}$ & (std.dev.) & $\begin{array}{l}\text { Spatial } \\
\text { (mean) }\end{array}$ & (std.dev.) \\
\hline $305 \mathrm{~nm}$ & $59.2 \%$ & $1.9 \%$ & $56.6 \%$ & $1.1 \%$ \\
$340 \mathrm{~nm}$ & $58.4 \%$ & & $56.1 \%$ & \\
$400 \mathrm{~nm}$ & $59.2 \%$ & & $55.7 \%$ & \\
$495 \mathrm{~nm}$ & $60.9 \%$ & $0.7 \%$ & $58.2 \%$ & (VIS2)
\end{tabular}

Table 2. Spectral and spatial MTF dependence at Nyquist Frequency

Although most of the results of an MTF derivation @ Nyquist are around $60 \%$, we had no single result above the $63 \%$ limit. This could be an indication of the correctness of the evaluation. There were also no abnormalities in intensity and spectral dependence.

The resulting detector MTF shall fulfils the following requirement over the full spectral range of the UVVIS CCD:

- In the $27.5 \mu m$ direction the (spatial) MTF shall be better $58 \% @$ Nyquist.

- In the $15.0 \mu m$ direction the (spectral) shall be better $45 \%$ @ Nyquist.

The MTF in the spatial direction almost meets the requirements. (The real results are between $56 \%$ and $58 \%$ ). However, the MTF in the spectral direction is clearly better than required. (Between $58 \%$ and $61 \%$.)

Open issues are related with

- the analysis of asymmetries and conspicuously structure on measured signal form and the influence on the MTF and,

- the effect that the MTF in the spatial direction is $2 \%-3 \%$ smaller than in the spectral direction.

\section{ACKNOWLEDGEMENTS}

The presented work has been performed under ESA contract. The authors would like to express their thanks to their respective colleagues at Airbus Defense and Space, ESA and EUMETSAT, and to all partner companies within the SENTINEL 4 industrial consortium for their valuable contributions to the continuing success of this very challenging program. This article has been produced with financial assistance of the EU. The views expressed herein shall not be taken to reflect the official opinion of the EU.

In addition, the authors would like to thank all members of the team at DLR and Airbus, but especially Mathias Uhlig, for their tremendous support in measuring and data processing. 


\section{REFERENCES}

Boreman, G. D., 2001. Modulation transfer function in optical and electro-optical systems. Vol. 21, SPIE press Bellingham, WA.

Jahn, H. and Reulke, R., 2009. Systemtheoretische Grundlagen optoelektronischer Sensoren. John Wiley Sons.

Jerram, P. and Morris, D., 2016. Recent sensor designs for earth observation. Vol. 9881, pp. 988111-988111-14.

Lesser, M., 2015a. A summary of charge-coupled devices for astronomy. Publications of the Astronomical Society of the Pacific 127(957), pp. 1097.

Lesser, M., 2015b. A summary of charge-coupled devices for astronomy. Publications of the Astronomical Society of the Pacific 127(957), pp. 1097.

Reulke, R., Brunn, A. and Weichelt, H., 2011. Spatial resolution assessment from real image data.

Swindells, I., Wheeler, R., Darby, S., Bowring, S., Burt, D., Bell, R., Duvet, L., Walton, D. and Cole, R., 2014. Mtf and psf measurements of the ccd273-84 detector for the euclid visible channel. Vol. 9143, pp. 91432V-91432V-8. 